\title{
An interactive environment for teaching a watch crew to act properly in case of wind and wave perturbation
}

\author{
Nelly Sedova ${ }^{1}$, Viktor Sedov $^{2}$, Ruslan Bazhenov $^{3}$, Dmitry Luchaninov $^{4}$, Vladimir Glagolev $^{5}$ \\ ${ }^{1,2}$ Maritime State University named after G. I. Nevelskoy, Vladivostok, Russia \\ ${ }^{3,4,5}$ Sholom-Aleichem Priamursky State University, Birobidzhan, Russia \\ ${ }^{3}$ Corresponding author

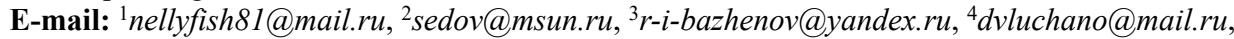 \\ 5glagolev-jar@yandex.ru
}

Received 13 March 2019; accepted 31 March 2019

DOI https://doi.org/10.21595/vp.2019.20653

Check for updates

Copyright $(2019$ Nelly Sedova, et al. This is an open access article distributed under the Creative Commons Attribution License, which permits unrestricted use, distribution, and reproduction in any medium, provided the original work is properly cited.

\begin{abstract}
An interactive environment is supposed to be one of the most important technologies for developing teaching systems for a deck crew. It enables modeling different interworking scenarios (excursions) among marine crafts as well as responding maritime facilities to operator performance, etc. The ship crew is possible to be taught in order to know how to act effectively in alarm or challenging situations in such an environment. A virtual polygon can also be used as a decision making support system to forecast growing threats and then choose the best behavior strategy/policy. The paper illustrates the findings of a virtual polygon development for simulating navigation safety problems with Blender environment applied for a three-dimensional graphic image representation, Delphi programming language for software support of the developed methods and a user interface design. The authors explained a layout algorithm for constructing three-dimensional offshore surface vessels. Moreover, the researchers simulated various situations in different weather effects and wind and wave perturbation in their study.
\end{abstract}

Keywords: virtual polygon, navigation safety, emergency situations at sea, simulation, navigator simulator, teaching a watch crew, wind and wave perturbation.

\section{Introduction}

Virtual polygons established for navigation allow one to test prototypes of above-water crafts movements one to another, to other crafts, such as low-flyers, and the environment/ external conditions. One can simulate challenges in blind navigation, as well as in emergency or pre-emergency mode in the virtual proving ground. In virtual training areas, it is possible to model situations when it is impossible to avoid ship collisions. In this case a virtual polygon is able to predict the result of several conclusive result options and estimate the best / worst ones, which allows one to use a virtual polygon for decision making systems for navigators. Moreover, the necessary calculations are performed online, so using virtual polygons is recommended in onboard decision making systems.

That is why many researchers have an attempt to develop virtual polygons to test prototypes of various air-sea situations. The authors refer to several scientific papers related to the focused subject matter either in a direct or indirect way.

The best of the current educational systems contains models for typical situations that happen on a shipboard or at sea [1]. Furthermore, many of them involve high-quality visualization of the designed situation according to 3D modeling technologies, virtual and/or augmented reality. For example, paper [2] deals with a training platform for network marine learning, which is 3D virtual network environment with the capability to simulate different situations, while the platform combines e-learning methods and techniques and distributed virtual environment technologies. The paper presents the authors' findings on 3D modeling, developing 3D spaces and the interaction of $3 \mathrm{D}$ images. The paper notes that the critical technology for designing marine training systems is an interactive marine environment in virtual reality and online mode. Though, the authors emphasize a number of drawbacks connected with 3D multi-user online Internet training 
caused by a low bandwidth (network) and high network delay. Some methods that provide the elimination of these shortcomings are applied.

The paper [3] also proposes a marine simulator through a virtual reality technology based on low-cost and easy-to-maintain Desktop Marine Teaching systems. The same paper gives the results of combining FMMS (Full Mission Marine Simulator), DMTS (Desktop Marine Teaching System) and WMTE (Web-based Marine Training Environment) technologies [2] in a united multi-level virtual reality system for training sailors in all maritime training activities.

The authors of the paper [4] give an example of container anchorage terminals to offer two simulators for training port managers. One of them is designed to model a straddle carrier, and the other one is for simulating a truck operation. Both of these simulators use the same virtual environment that reconstructs Gioia Tauro container anchorage terminal. The paper [5] proposes a marine vessel simulator and traffic control service simulator using a united virtual environment for training port managers. The work [6] is devoted to the development of a simulator for controlling a vessel. In order to increase its proximity to real situations, the simulator integrates real items into the modeling process. The information about them is obtained from an Automatic Identification System (AIS). In this regard, the authors dwell on a number of additional practical problems arising in developing the architecture of the necessary software.

It is known from the paper [7] that simulators are used in education to imitate a ship engine operation in a virtual reality. The simulator includes several modules: a module with a three-dimensional model of a marine engine, a module for an automatic assessment of students' skills, a module containing a test system, as well as a module of a gallery of questions and a module providing communication between the simulator and the examination center, which can estimate the crew functioning by several simulators. The authors use an algorithm based on fuzzy logic to assess the crew performance in simulated virtual conditions. In paper [8] the authors use 3D-Max and visualization with V-ray to develop the engine room simulator. The developed simulator has quite many mechanisms to interact with. Besides, the module for an automatic assessment of students' competence is based on the fuzzy set theory as in paper [7]. Thus, it makes evaluating the knowledge level more frank.

It is also important to point out that there is an opportunity to train the crew to act in such emergency situations that cannot be performed in real conditions according to safety requirements for precisely that reason for using simulators [9].

\section{The structure of the virtual polygon to model safe navigation problems}

The developed virtual polygon for modeling safe navigation problems includes the following software components: a subsystem of graphic images, a warning (sound) subsystem, a simulation subsystem, an intelligent subsystem, a graphical user interface configuration subsystem.

The subsystem of graphic images allows visualizing sea surface crafts, stationary or dynamic items that can potentially be found along the sea vessel course, various models of wind and wave disturbances and sea surfaces.

The sound (alarm) subsystem provides sound signals to prevent the emergency situations. The subsystem includes some extra several sound effects of hydrometeorological phenomena for the simulation modes.

The simulation subsystem is intended to generate various situations that happen at sea, for example, situations of two ship collision approach, cases of ship collision avoidance with stationary unmanned crafts, etc.

The intelligent subsystem includes a number of intelligent models for making decisions in various situations. For example, one of the models based on neural network technologies and the fuzzy set theory provides a decision-making process for ship collision avoidance, whether another fuzzy model is used for ship collision avoidance with stationary unmanned crafts.

The graphical user interface configuration subsystem is designed to provide convenient interaction between the virtual polygon and a user. 


\section{The subsystem of graphic images}

The graphics image subsystem contains three-dimensional models of several kinds of guided and unmanaged crafts: sea surface boats (for example, such sea vessels models as small fishing boats, tankers, sailing ships, etc.); stationary constructions (markers drogues/, radar approach beacons, coastal zones, etc. .); dynamic scopes (drifters, low-flying quadcopters, etc.); various meteorological conditions (W'r), for example, blind navigation; sea surfaces with visual representation of the wind afteraction influencing different scopes and commotion when the wind agitates the sea or when the sea is still in the wave crest-shape, spindrift, etc. An available free-of-charge editor of three-dimensional modeling Blender was used to visualize graphic images. Blender editor has a user-friendly graphical interface supplied by manageable functions enabled from a keyboard.

The authors would like to outline the algorithm for designing such a three-dimensional model of a marine vessel as a small fishing boat type (SFB) To begin with, it should be noted that the model was designed for a real craft having the following dimensional characters: length $-16 \mathrm{~m}$, width $-4 \mathrm{~m}$, bow draft $-0.8 \mathrm{~m}$, draught aft $-1.6 \mathrm{~m}$, capacity of craft -21 tones, speed -8 knots, engine output $-165 \mathrm{CV}$. The model of this sea vessel was chosen on purpose. Full level tests were carried out on this particular board for a certain part of intelligent systems that were included in the intelligent subsystem of the virtual polygon.

3D model of SFB consists of two main separate parts. These are a skeleton and a back of the deck. The skeleton contains a marine hull, a crew cabin, a deck, upper works of the after-part, and small elements of the watercraft hull. The back of the deck includes the under-body of the after-part: a back of the deck, a rudder and a screw bolt. The first step in constructing a 3D model of the SFB is to extrude the marine hull from a primitive object of a cube type. 'Extruding' is a built-in feature in the 3D modeling environment that enables to put the object a desired shape. The primitive object 'cube' is divided into many cube faces. The specified faces are extruded in different directions along $X, Y, Z$ axes, forming the vessel hull. The vessel hull is lined out lots of small blocks, forming the future vessel components.

The second step in developing a 3D model of SFB is to extrude such an element of SFB as a crew cabin. The cabin of SFB was extruded up along the $\mathrm{Z}$ axis from the marked ship hull surface that had been extruded before. The crew cabin of SFB was also lined out lots of small blocks. Port-lights and a wedge-action valve were made according to the markdown.

The third step in constructing 3D model of SFB is to complete the vessel skeleton by "building up the sides". Building up the sides are carried out by pulling up (along the $Z$ axis) the intended area on the vessel hull (deck). Setting up lifelines/ guard railings and small elements of the deck is carried out from standard primitive objects, i.e. by editing and superimposing them on a ship.

The fourth step of developing 3D model of SFB involves the second main element of the vessel back of the deck (stern) modeling. The designing of a stern is similar to that of a skeleton from a primitive object of the cube type. The stern in 3D model of SFB refers to the whole underwater part of the stern, containing the following elements of the vessel: a stern, a rudder plate and a screw bolt.

The fifth step of designing 3D model of SFB is to link the two main elements: a skeleton and a stern. They are connected by snapping in two parts together.

In the sixth step of developing 3D model of SFB, small details are included in the model of SFB, such as afterdeck parts (a table, benches), a life ring buoy, etc.

It the seventh (final) step of producing 3D model of SFB, the authors carry out texturing of certain faces of the vessel onto the color space (texture map) by the projection method. This is how each element of SFB got its own structure: the color and feature.

The authors develop corresponding 3D scenes to visualize sea surfaces. They modeled them by setting three surfaces (planes) in space: a horizontal one for direct imaging of the surface and two vertical ones for taking a background image. The color spaces were produced with the Paint graphic editor, and two types of textures were developed: structures of the sea surface and those 
of the background. At the first stage, the blanks were made, the auto screen blanks of $1024 \times 1024$ and $1024 \times 512$ pixels. The billets of the first size are used for taking a picture of the sea surface; the second one is in charge of the background image. The next stage was to intend the corresponding pattern in the blanks. Each 3D scene was performed in a proper graphic palette of spaces (textures). The sea was visualized on a horizontal plane by applying a wave modifier embedded in Blender graphics editor capable of transforming the plane into a body wave (Fig. 1).

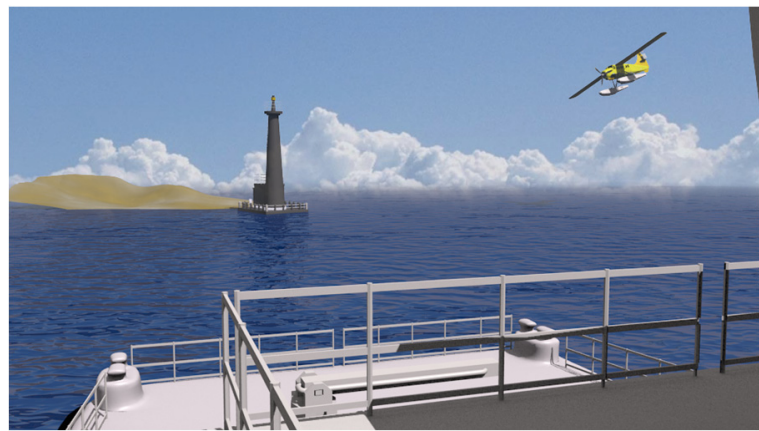

Fig. 1. The effect of rendering the finished composition to the person standing on SFB bridge

\section{Simulation subsystem}

Each situation listed in the simulation subsystem consists of offshore surface crafts models contained in the subsystem of graphic images, and a staged environment that included sea surfaces in different meteorological conditions and the presence / absence of different guided and unmanned crafts.

Delphi development environment is used to design the graphical user interface of the simulation modeling subsystem. A graphical user interface for modeling various situations mentioned in the Beaufort wind force scale is a form with thirteen buttons, an area for outputting a situational pattern, and an output field for a verbal definition of wind capacity, an average wind velocity, and a description of the wind effect in the main (open) sea. The description contains the information on various situational patterns, displayed in a graphical user interface.

\section{Intelligent subsystem}

The intelligent subsystem of the virtual polygon for simulating navigation safety tasks contains the following intelligent models: an intelligent model of ship collision avoidance based on the fuzzy set theory, an intelligent model of ship collision avoidance based on neural network technologies and the fuzzy set theory, a fuzzy model of ship collision avoidance with stationary unmanaged crafts, a fuzzy model for assessing the hazard level of marine casualties, etc.

Among other factors, a model based on the fuzzy set theory that determines the emergency level automatically according to the information issued in the simulation subsystem. Such a model is capable to determine one of the four emergency levels (incident, major incident, emergency, extreme emergency) according to the amount of harm injuring a person / a group of people's health and / or life, the extent of the marine vessel damage, as well as the amount of environmental pollution) [10]. The authors published the outcomes of their review of test situations in paper [10]. They confirm the efficiency of the proposed model for determining the emergency level. Thus, there is a comparison of the effects of testing a fuzzy model that determines the emergency level. It shows the values below: Mean Absolute Error $(\mathrm{MAE})=3.319 \times 10^{-1}$, Root Mean Square Error $(\mathrm{RMSE})=5.179 \times 10^{-1}$, Symmetric Mean Absolute Percentage Error $($ SMAPE $)=2.49 \times 10^{-2}$.

A user enters the information on how many victims (injured, gone missing or died) were in a virtual polygon through a graphical user interface (GUI). A user also enters the information on constructive a vessel hull damage, as not causing navigability wastage, and on the contrary, 
leading to the wreck of a ship or its complete functional wrecking. It can be featured by the navigability wastage. A user can also enter the data on environmental pollution, qualified by the scope of spilled petroleum products (in tons). According to the intelligent subsystem, an emergency level value is generated in the simulation subsystem. It is displayed on GUI of the virtual polygon.

To simulate the problems of avoidance of two ship collisions, the information on bearing, seaways and sea craft velocity is used. They require making a decision on collision avoidance. Fuzzification and a fuzzy inference algorithm are carried out for each of these parameters. There are ground sets, on which the linguistic variables (LV) of the intelligent model of collision avoidance are determined. They are set as the following: input LV bearing, a vessel-operator seaway and a target-vessel seaway run through the set of values $[0 ; 360]^{\circ}$. The set of values for the input LV target-vessel velocity is determined according to the information obtained as a result of information analysis from The Russian Maritime Register of Shipping (RS) - $[0 ; 34]$ knots. The set of values that runs through the output LV corresponds to the segment $\left[-60^{\circ} ; 360^{\circ}\right]$. The value $-60^{\circ}$ corresponds to the term hard left, the value $-30^{\circ}$ means the term to the left, and the value $0^{\circ}$ corresponds to the term right ahead (neither operator vessel seaway, nor speed changes), $60^{\circ}$ stands for hard right, $30^{\circ}$ means to the right, and $360^{\circ}$ is a turning circle maneuver (respectively, the term turning circle). The intelligent subsystem contains a modified version of the intelligent ship collision avoidance system [11]. The modified version of the intelligent model of collision avoidance includes the input LVs mentioned earlier. The group of navigators did an expert survey and obtained the values for the outputs. Thus, a training sample was generated. It consists of 525 elements. As a result of computer simulation, the researchers identified Fuzzy Logic Toolbox, the optimal parameters of neural-fuzzy inference using Matlab math software. They are Adaptive Neuro-Fuzzy Inference System with grid generation by the lattice method without clustering. The scholars used the hybrid method as a back propagation of error algorithm combined with the least-squares method. The product of two sigmoid membership functions was used as the membership function. The best neuro-fuzzy network was trained, which solves the problem of collision avoidance best of all. The specified neuro-fuzzy model, introduced into the intelligent subsystem of the virtual polygon, returns test results equal to MAE $=1.165 \times 10^{-5}$, $\mathrm{RMSE}=6.063 \times 10^{-5}, \mathrm{SMAPE}=6.2555 \times 10^{-7}$. Modeling of the neuro-fuzzy network has proved that the number of learning stages more than 100 does not affect the learning quality greatly.

There are two opportunities to generate the current situation for the problem of ship collision avoidance in the virtual polygon simulation subsystem. On the one hand, a user can specify the necessary values for bearing, seaways and speed of two sea vessels participating in situation modeling by GUI by himself. On the other hand, the parameters of the generated situation are determined automatically in random manner. In both cases, the current situation is generated in the virtual polygon. The location of two sea vessels is visualized considering the parameters. Then, the information is requested from the intelligent subsystem that recommends the vessel to perform the necessary maneuver. Also, the user can be specified through GUI whether to continue the situation development according to the adopted intelligent collision system value or not. If there is a positive rating, sea ships moving along their new seaway or seaways are displayed.

\section{Conclusions}

To conclude, the authors' finding of the studies in designing the virtual polygon for modeling safe navigation problems was a series of 3D offshore surface crafts. Moreover, the researchers simulated various situations taking into account different weather conditions. At present, the authors keep on enhancing the capability features of the developed polygon, in particular, working on adding new guided or unmanaged crafts and various standard situations to the subsystem of graphic images and the subsystem of simulation modeling. Finally, the authors have much work on a set of situational patterns (digital prototypes) of intelligent navigation safety systems in progress. 


\section{References}

[1] Stan L. C., Buzbuchi N. The importance of the educational factor to assure the safe and security on the sea. International Journal on Marine Navigation and Safety of Sea Transportation, Vol. 3, Issue 3, 2009, p. 193-196.

[2] Xie C., Liu X., Jin Y. A prototype of the Web-based marine training environment. International Conference on Web-based Learning, 2005, p. 78-85.

[3] Xiuwen L., Cui X., Yicheng J. Multi-level virtual reality system for marine education and training. 1st International Workshop on Education Technology and Computer Science, Vol. 2, 2009, p. 1047-1050.

[4] Bruzzone A., Longo F., Nicoletti L., Diaz R. Virtual simulation for training in ports environments. Proceedings of the Summer Computer Simulation Conference, 2011, p. 235-242.

[5] Bruzzone A., Longo F., Nicoletti L., Diaz R. Traffic controllers and ships pilots training in marine ports environments Proceedings of the Symposium on Emerging Applications of M\&S in Industry and Academia Symposium, 2012, p. 61-68.

[6] Last P., Kroker M., Linsen L. Generating real-time objects for a bridge ship-handling simulator based on automatic identification system data. Simulation Modelling Practice and Theory, Vol. 72, 2017, p. 69-87.

[7] Sun J., Zhang Q., Huang D., Zhang L. A novel networked marine engine simulator for crew operation examination with auto evaluation using virtual reality technology. International Conference on Virtual Reality and Visualization, 2014.

[8] Shen H., Zhang J., Cao H. Research of marine engine room 3-D visual simulation system for the training of marine engineers. Journal of Applied Science and Engineering, Vol. 20, Issue 2, 2017, p. 229-242.

[9] Cwilewicz R., Tomczak L. Improvement of ship operational safety as a result of the application of virtual reality engine room simulators. WIT Transactions on Information and Communication Technologies, Vol. 39, 2008, p. 535-544.

[10] Sedova N. A., Sedov V. A., Bazhenov R. I. Analysis of emergency level at sea using fuzzy logic approaches. Advances in Intelligent Systems and Computing, Vol. 658, 2017, p. 314-322.

[11] Sedova N. A., Sedov V. A., Bazhenov R. I. The neural-fuzzy approach as a way of preventing a maritime vessel accident in a heavy traffic zone. Advances in Fuzzy Systems, Vol. 2018, 2018, p. 2367096. 